\title{
Post-disaster Psychosocial Capacity Building for Women in a Chinese Rural Village
}

\author{
Timothy Sim ${ }^{1,2,3} \cdot$ Jocelyn Lau $^{1} \cdot \mathrm{Ke} \mathrm{Cui}^{3} \cdot \mathrm{Hsi}^{-H s i e n ~} \mathrm{Wei}^{4}$
}

Published online: 20 May 2019

(C) The Author(s) 2019

\begin{abstract}
Mental health interventions following disasters have been criticized as individualistic, incomplete, and culturally insensitive. This article showcases the effects of a culturally relevant and sustainable psychosocial capacitybuilding project at the epicenter of the 2008 Wenchuan Earthquake. Specifically, the project focuses on women, a group that has received limited attention in post-disaster recovery in China. This qualitative research study $(N=14)$ sheds light on the characteristics and processes of the implementation of a post-disaster psychosocial intervention project in rural China. In addition, by adopting the Success Case Method as an evaluation approach, this study elucidates its effects on the psychological and social changes of the disaster victims. The findings capture five aspects of psychosocial changes: enriched daily life, better mood, enhanced self-confidence, increased willingness to socialize, and the provision of mutual help. This study hopes to encourage more culturally relevant and empowering practices for women in building their psychosocial capacity after disasters.
\end{abstract}

Jocelyn Lau

jocelynlau.lau@gmail.com

1 Department of Applied Social Sciences, The Hong Kong Polytechnic University, Hong Kong, China

2 World Health Organization Collaborating Centre for Community Health Services, School of Nursing, The Hong Kong Polytechnic University, Hong Kong, China

3 School of Public Administration, Sichuan University, Chengdu 610065, China

4 Department of Building and Real Estate, The Hong Kong Polytechnic University, Hong Kong, China
Keywords China $\cdot$ Natural hazard-induced disasters $\cdot$ Post-disaster recovery $\cdot$ Psychosocial capacity building · Success case method - Women' empowerment

\section{Introduction}

Disaster vulnerability is complex; it captures a combination of characteristics of the individual, group, or community, as well as the impact of social, economic, cultural, and political factors. All of these variables affect one's ability to anticipate, cope with, and recover from a disaster (Wisner et al. 2004; Gil-Rivas and Kilmer 2016). Among survivors, women have been identified as one of the most vulnerable groups during and after disasters, due to their physical vulnerability and disadvantaged position in many societies (Aryal 2014; Baker and Cormier 2014; Sohrabizadeh et al. 2016). In addition to dealing with the consequences of the event itself, they are usually further burdened or weakened after a disaster, as women are expected to carry out normal domestic tasks despite the more challenging and complex post-disaster environment (Twigg 2004).

On 12 May 2008, an earthquake with 8.0-magnitude occurred in Wenchuan County, Sichuan Province, China. It was the deadliest and strongest one to hit the country since the 1976 Tangshan Earthquake. The catastrophe left more than 87,000 people dead or missing and more than 374,640 injured in Sichuan and the neighboring provinces (Liu 2009). Moreover, the earthquake deprived 1.15 million rural people in Sichuan Province of their livelihood, as the tremors wreaked havoc on their leased farmland and forests (Rao et al. 2011). These factors impacted residents in devastated areas adversely in terms of health, property, housing, employment, and their living environment. In 
addition, the earthquake was not only a natural calamity but also a cultural catastrophe; it hit areas in where the majority of Qiang and Zang (Tibetan) ethnic minority groups resided, and destroyed most of the area's precious cultural relics (Han et al. 2016).

The earthquake had seriously disrupted many women's social support networks as a result of the severe loss of close family members, colleagues, friends, and neighbors. It created a huge shock to local women's social lives and self-identities in a rural and ethnic diverse setting ( $\mathrm{Hu}$ 2010). Many female disaster victims reported recurrent symptoms of crying, sleep problems, and suicidal ideation after the 2008 earthquake (Li et al. 2011). Although many nongovernmental organizations provided mental health and psychological support to survivors, most of their attention directed towards children, adolescents, and older people, with little attention paid to women (Huang and Wong 2013). In light of this, a psychosocial capacity-building project that aimed at enhancing the psychosocial well-being of women survivors was set up in the epicenter, and this study reports on the development and insights of the effect of the project initiated in a rural Chinese village located at Yingxiu Town, the epicenter of the 2008 Wenchuan Earthquake. Specifically, it emphases the importance of promoting culturally relevant and empowering practices for women, without undermining their resilience, as well as their significant contributions to their families and communities following disasters.

\section{Mental Health and Psychosocial Support}

Mental health and psychosocial support have increasingly been seen as a standard humanitarian response to the negative effects of disasters (Tol and van Ommeren 2012). Although mental health protocols and psychosocial support are often applied complementarily, their nature of focuses are in fact fundamentally different. The Inter-Agency Standing Committee (IASC 2007) reiterated that the focus of mental health protocols is more on the psychological consequences of disaster victims-emphasizing disaster survivors' stress and reaction to trauma, particularly in terms of post-traumatic stress disorders. In contrast, psychosocial support pays greater attention to the social aspects of recovery and considers psychosocial strategy as an effective mental health care (Becker 2007; Suarez 2016). Its emergence was in part a reaction against the protocols' overemphasis on the individual at the expense of collectivity and the community (IASC 2007; Miller 2012).

In his seminal work, Psychosocial Capacity Building in Response to Disasters, Miller (2012) was one of the first to propose a unified model of post-disaster psychosocial capacity building. He defined psychosocial capacity building as "intervention, provided by professional and non-professional people, both local and from the outside, that constitutes a multi-systemic, culturally grounded, empowerment- and resiliency-oriented approach designed to help individuals, families, social groups, and communities recover from a disaster. Psychosocial capacity building seeks to be sustainable over time and builds on the foundation of local capacities and resources" (Miller 2012, p. 191).

The definition itself reveals four foundational principles of the psychosocial capacity building (PCB) model: (1) cultural responsiveness must be a key in the process of psychosocial capacity building; (2) collective capacity should be rebuilt through empowering local people; (3) individuals' strengths and sources of resilience should be at the forefront; and (2) families, social groups, and communities should be seen as the core units of psychosocial rebuilding following a disaster.

These underlying principles reinforce the differentiation of the approach from one that solely focuses on mental health recovery to an ecological psychosocial one that considers more than the individual and places greater emphasis on rebuilding and strengthening collective capacity (Miller 2012).

\section{Project Planning, Design, and Intervention}

The target group of this project was women disaster victims from Zhangjiaping Village of Yingxiu Town, which was the epicenter of the Wenchuan Earthquake that occurred on 12 May 2008. The village is situated in Wenchuan County, where is predominantly inhabited by people of the Qiang ethnic group. The impact of the quake on Yingxiu Town alone could be reflected in number terms - out of the original population of about 15,000 in the town, there were only 5000 left after that fateful quake (Siu 2016). What is worse, the quake triggered numerous natural hazards, namely, landslides and debris flows, which continued to afflict local population with chronic mental pressure in the years following the catastrophe. Especially during the wet season, the loose soil and rock materials distributed on steep slopes and in channels caused by the quake could easily evolve into debris flows once triggered by heavy rainfall (Xu et al. 2012). The intensity and frequency of these natural hazards were consistently high. For instance, the heavy rainfall on 24 September 2008 triggered 72 debris flows in Beichuan County (Tang et al. 2011), where the old county town of the epicenter was completely buried by 6 to $10 \mathrm{~m}$ thick debris. Another rainfall on 13-14 August 2010 caused 80 debris flows in Qingping, Yingxiu, and Longchi Towns, leaving the reconstructed towns partially or totally destroyed by the debris flows (Huang and 
Li 2014). The severest one occurred on 9-11 July 2013 where the impacted areas covered more than $90 \%$ of communities in Wenchuan County. Particularly, over 240 debris flows occurred on 10 July alone along the Min River and its tributaries (Ge et al. 2015) in where Zhangjiaping Village is situated. In addition to casualties, a large number of buildings, factories, and even reconstructions were ruined. The mountainous traffics were severely interrupted every year that left so many towns isolated (Ge et al. 2015).

Against this background, as part of the post-disaster rebuilding initiatives, this social work project dedicated to the vulnerable group was designed to achieve three overarching objectives:

(1) Alleviate women's feelings of distress and increase their quality of life;

(2) Increase women's participation in community affairs; and

(3) Invigorate the community by enriching its cultural life and increasing its cohesion in the face of continuing disaster threats.

In addition to integrating the principles of Miller's (2012) PCB model into the research design, the project endorsed the use of group work to optimize the village's cultural resources and community capacity. The process of project development was guided by a step-by-step principle that was deemed practical in conducting post-disaster psychosocial work in China (Sim and Dominelli 2017). This principle stresses the need of social workers to be contextually respectful when working in volatile post-disaster situations.

Overall, the project development can be divided into five incremental phases, with some inevitable overlapping components: (1) form a natural group; (2) develop group cohesion; (3) perform tasks spontaneously; (4) promote community interaction; and (5) expand its effect through increased interaction with new group members and the larger community.

\subsection{Phase 1: Formation of a Natural Group (May- September 2014)}

Through ongoing observation and interaction, our social workers, who had been working in Zhangjiaping Village since 2008 (Sim 2009), learned that Guozhuang dance is a popular collective and ethnic folk dance-exercise that originated in the Aba Autonomous Prefecture (Liu et al. 2009). It is a regular form of exercise that can improve health and relieve stress (Zhang and Liu 2014). Suitable across age and gender, this cultural dance can serve as a platform to connect different groups of people within and in other villages ( $\mathrm{Li} 2007$; Liu and Chen 2010). Moreover, there are readily available local resources, namely cultural expertise, music, and costumes, which made Guozhuang dance an obvious and favorable choice when developing a project that has the potential to be the nexus for community sustainability.

The key actor in this PCB project was a social worker of Qiang ethnicity. Before qualifying as a social worker, he was a professional dance trainer. Because of his ethnic descent, our colleague was able to understand the cultural rituals and the Guozhuang dance movements. He used his professional social work values, knowledge, and skills (for example, project planning and budgeting, as well as group development dynamics and techniques) to build a strong rapport with different groups of people in the village.

In May 2014, the cultural dance project, which was intended to promote the psychosocial well-being of village women, was officially initiated with the launch of an informal Guozhuang dance class in Zhangjiaping Village. Over the next 4 months, a 1-h session was held five times a week in the central square of the village. The dance class usually commenced after dinner. Led by the social worker, it began with some warm-up exercises, followed by practicing various dance movements, and wrapped up with short relaxation exercises and a debriefing.

During this phase, the social worker served as a facilitator to exchange knowledge and skills with the local people. He played a vital role in reaching out proactively and devising different strategies to motivate village women's interest in participating in the dance class, and by mobilizing active participants to invite their fellow village women. Emboldened by their peers, neighbors, and friends, the dance class steadily increased in the number of women participants over time, and an informal dance group was eventually formed. Despite an absence of a systemic structure, the emergence of an informal dance group could be understood as the "forming" stage of a natural group identified by Tuckman and Jensen (1977).

\subsection{Phase 2: Development of Group Cohesion (July- August 2014)}

As the dance class continued to evolve, the social worker detected conflicts among its members. The emergence of intragroup conflicts (Bonebright 2010) signalled the beginning of the "storming" stage of group development. To lead the group into the "norming" stage, where group norms are resolved and the formation of trust is apparent (Braaten 1974/1975), the social worker focused on promoting group cohesion by using group facilitation skills and group dynamics. Specifically, the social worker designed and organized five sessions of team-building activities over a 5-week period. The goal was to work on members' readiness to stick together in subgroups (Carron et al. 1998). Through various indoor team games and group 
exercises that facilitate communication and socialization, the village women were encouraged to reflect on and exchange their feelings during the process. In addition, a 2-day outdoor team-building camp was organized. The objectives were mainly to (1) help release tensions and distress among members; and (2) provide them with an opportunity to explore their roles in the village and to set goals for the dance group in the future.

\subsection{Phase 3: Spontaneous Performance of Tasks (August-September 2014)}

Gradually, the women from Zhangjiaping Village began to gather and dance spontaneously every evening. As time went by, their impromptu gathering for Guozhuang dance practice became a daily routine, as well as an opportunity to exercise, relax, and socialize. Furthermore, with the encouragement of the social worker, the women extended the invitation to other villagers, particularly children and the elderly, who were keen to participate in this cultural practice. It is apparent that the woman group became a nexus for families and community members of the Village. While the facilitative role of the social worker began to recede from this point onward, the dance group was poised to form a formal dance team to further promote the well-being and mutual support among village residents. On 30 September 2014, a ceremony was officiated by the village chief to establish the Guozhuang dance team formally. The ceremony featured as a villagewide gala with cultural performances and games that involved almost everyone in the community. This showcased the collective effort of the village women in collaboration with the professional social work team.

Formalization of a more organized, purposive, and autonomous group demonstrated the women's strengths and resources and affirmed their resilience as an essential element of psychosocial rebuilding following a disaster. It was the first time the women of Zhangjiaping Village appeared and performed as a team at a formal occasion, which was a ground-breaking experience not only for this group themselves but also for the village as a whole. During the ceremony, the village chief and village party secretary were also present to express appreciation and support for the dance team. In the months that followed, the Guozhuang dance team performed on a variety of public occasions, including an invitation from the Yingxiu Town government. This further enhanced the confidence of individual village women and them as a collective unit.

\subsection{Phase 4: Promotion of Community Interaction (October-December 2014)}

Apart from being actively involved in the Guozhuang dance, the women from Zhangjiaping Village began to organize and invite others to participate in a variety of other activities during special occasions. They engaged other groups and community members, including the village committee, children, and older people, and acted as catalysts in connecting these diverse age groups with one another. For example, on one mid-autumn day, the dance team invited other women to cook for all of the elderly as a way to express gratitude and care for the senior.

Moreover, the dance group became a hub to initiate and provide services to the community, such as offering Guozhuang dance classes to older people in the evening. Through teaching and sharing, the women were given opportunities to demonstrate their ability to create a vibrant and friendly environment in and for the community. The gradual development of the group augmented the psychosocial well-being of the families, social groups, and communities of the village.

\subsection{Phase 5: Expansion of Positive Impact (January-May 2016)}

By May 2016, the original dance group of 14 women from Zhangjiaping Village expanded its membership to 105 women from seven other villages of Yingxiu Town. A snowball effect of the cultural dance project was observed, with women from one village proactively inviting women from other villages to participate in the Guozhuang dance class. In view of the growing number of Guozhuang enthusiasts, a Yingxiu Town dance center was established by the Wenchuan County government; this facility provided an office and activity space in which the women could operate. Funding for the dance center was also allocated in view of the significant effect of the project on the local community.

In a nutshell, the project achieved its aim to empower village women to proactively take care of their well-being. The PCB project was cautious to involve women not as passive service recipients, but as participants, actors, and decision makers, who were capable of providing services to enhance and promote not only their own well-being, but also that of others in the community. Once the women took over the leadership role, the professional social work team gradually retreated, and eventually diminished to merely present to provide guidance to team leaders when necessary. This empowered the village women to be independent in order to sustain the established work and connections even when the social workers were fully withdrawn from the field.

\section{Evaluation of the Project}

This study was conducted immediately after the fourth phase, in which the women in Zhangjiaping Village began promoting community interactions. This timeframe was 
chosen for evaluation because confirmation of the impact of the project was deemed necessary before commencing the next phase where Guozhuang dance project was to be introduced to other villages of Yingxiu Town.

\subsection{Methods}

Employing Brinkerhoff's (2005) Success Case Method (SCM), we adapted it in such a way to fit into local conditions to examine the characteristics that the village women changed through participating in this cultural dance project rooted in Zhangjiaping Village. The challenges on researching time and population in conducting post-disaster fieldworks are commonly noted by researchers. Due to the unpredictable nature of disasters (Powell and Leytham 2014) and complications in disaster management, researchers often face difficulties to access to the field, which is an area often significantly politicized especially in developing countries (Olson 2000; Tierney 2007). Also, given that disaster research is always post hoc and reactive, the environment for data collection is featured with uncertainty, vulnerability, and complexity (Cutter 1996) than controlled and structured as appeared in traditional academic research. In addition, the target research populations, be it women, children, the elderly, the poor, minority groups, the injured, and even aid workers, would all become vulnerable psychologically and physically, to a certain extent, after a disaster (Hu 2015).

Considering the above unique post-disaster characteristics, this research was no exception but encountered difficulties in establishing a pretest and control group. The SCM was therefore considered as an alternative, when quasi-experimental or randomized experimental designs become unethical or unfeasible, or when evaluators have difficulty accessing equivalent and nonequivalent comparisons (Coryn et al. 2009), which was the case for this research. This study was approved by the Human Subjects Ethics Application Review Systems (HSEARS) research ethics committees of The Hong Kong Polytechnic University.

Conventionally, the SCM is a two-part process. The first part entails locating individuals who apparently have been successful (or unsuccessful) in learning and using the knowledge or skills acquired from attending training courses. This is often accomplished through a structured survey questionnaire with predetermined outcomes of interest based on previous evidence. The second part involves interviewing a sample of successful (or unsuccessful) participants and documenting their stories to provide in-depth insights into their lived experiences of change. Compelling success stories with persuasive details of real-life knowledge have been identified as an efficient tool to convey how a program was operated and succeeded
(Lewis et al. 2004). Most importantly, this method allows the capturing of specifics that cannot be conveyed by numbers, thus provides a greater width and depth of description on the context for readers who are unfamiliar with the program and the perplexing background to follow (Kibel 1999).

In our evaluation, we conducted a focus group study before the two-step SCM process. This was deemed indispensable because there is limited relevant evidence regarding psychosocial capacity-building programs for vulnerable groups, particularly for women, following disasters. This additional step enabled researchers to identify the strengths and weaknesses of the PCB project in accordance to the women's perspective on concepts and ideas they considered as relevant and important (Scott and Proescholdbell 2009). We will further elaborate the process in the "Data Collection and Analyses" section.

\subsection{Participants}

The data of this study $\left(N_{1}=14\right)$ were drawn from women who resided at Zhangjiaping Village in Yingxiu Town, the epicenter of the 2008 earthquake. A purposive sampling was conducted on women who were either directly or indirectly affected by the catastrophe. This study engaged women who were once the service recipients of the psychosocial capacity-building project to take part in the focus group interviews and structured survey on a voluntary basis. Considering the sensitivity and ethical concerns of the discussion of individuals' traumatic disaster experiences, as well as the unique "relation-oriented" nature of Chinese culture in social interactions (Cui 2015), the research design prompted the importance of pre-existed relationships between researcher and the research participants. Subsequently, based on the survey results, four of the participants $\left(N_{2}=4\right)$ were selected to participate in individual interviews. They were found to be the "successful cases" based on their highest scoring on the overall change in psychosocial well-being as reported in the questionnaire (Table 2). Given their informed consent, we presented the demographic characteristics of the participants $\left(N_{1}=14\right)$ in Table 1 .

\subsection{Data Collection and Analyses}

Two researchers who were unfamiliar with the participants collected and analyzed the data. The social worker involved in this psychosocial capacity-building project was also refrained from involving in the research process in order to avoid possible bias and generating socially desirable responses from the participants (Cui 2015).

As we indicated earlier, this study adopted Brinkerhoff's (2005) Success Case Method with deliberate adjustments in 
Table 1 Sociodemographic background of the participants $\left(N_{1}=14\right)$ in a psychosocial capacity-building study in Zhangjiaping Village, Yingxiu Town, Wenchuan County, Sichuan Province, China

\begin{tabular}{|c|c|c|c|c|c|c|c|}
\hline No. & Age & Ethnicity & Education level & $\begin{array}{l}\text { No. of } \\
\text { children }\end{array}$ & $\begin{array}{l}\text { Source of } \\
\text { income }\end{array}$ & $\begin{array}{l}\text { Husband away from } \\
\text { home } 2 / 3 \text { of the year }\end{array}$ & $\begin{array}{l}\text { Family loss and } \\
\text { injury in disasters }\end{array}$ \\
\hline 1 & 27 & Han & Elementary school & 2 & Farming & No & Yes \\
\hline 2 & 38 & Han & Secondary school & 1 & None & No & Yes \\
\hline 3 & 41 & Han & Elementary school & 2 & Wage income & Yes & No \\
\hline 4 & 27 & Han & Elementary school & 3 & Wage income & No & No \\
\hline 5 & 35 & Qiang & Elementary school & 2 & Wage income & No & Yes \\
\hline 6 & $27^{\mathrm{a}}$ & Han & Secondary school & 2 & None & No & Yes \\
\hline 7 & 31 & Han & Secondary school & 1 & None & No & Yes \\
\hline 8 & 41 & Han & Elementary school & 3 & None & No & Yes \\
\hline 9 & 27 & Qiang & Secondary school & 1 & Wage income & Yes & No \\
\hline 10 & $26^{\mathrm{a}}$ & Qiang & Secondary school & 2 & Wage income & Yes & No \\
\hline 11 & $32^{\mathrm{a}}$ & Qiang & Elementary school & 2 & Wage income & Yes & Yes \\
\hline 12 & $33^{\mathrm{a}}$ & Han & Elementary school & 2 & Wage income & Yes & Yes \\
\hline 13 & 39 & Qiang & Elementary school & 1 & None & Yes & No \\
\hline 14 & 28 & Han & Secondary school & 2 & Wage income & Yes & Yes \\
\hline
\end{tabular}

${ }^{a}$ Indicates the four women who participated in the in-depth individual interviews

${ }^{\mathrm{b}}$ Refers to disasters including the 2008 Wenchuan Earthquake and a catastrophic debris flow occurred on 9-11 July 2013

this research. The modified SCM began with a 1-h focus group interview conducted on all the 14 participants in two separate groups, with seven participants in each group. The key objective of the group interview was to explore the features of change, if any, of the participants ever since they started attending to the project's events and activities. The participants were encouraged to talk about similarities and differences of any psychosocial conditions they observed and went through. With their informed consent, the interviews were audio recorded. The audiotaped sessions were transcribed immediately after the focus group interviews. By means of thematic content analysis (Leedy and Ormrod 2005), open coding and axial coding were used independently by the researchers to analyze and categorize key words or sentences. The results were then inter-rated in order to increase the trustworthiness by ensuring its inter-rater reliability (Shenton 2004). Inconsistencies and disagreements were discussed among the two researchers. Agreement was later established through a consensus reached between the researchers and the authors.

Based on the themes identified in the focus groups, a survey questionnaire was then designed (available upon request) to ascertain the participants' demographic information, to measure the impact of the interventions, and to investigate the extent of psychosocial changes perceived by the participants. A set of questionnaire was distributed to each of the participants, which took them an average of $10 \mathrm{~min}$ to complete independently in a private environment. Each question consists of five options. Participants were asked to choose the one that best describes their psychosocial conditions. The five options could be converted to a Likert scale, where (A) Very much, signifying the highest score of 5; (B) Much, a score of 4; (C) A little bit, a score of 3; (D) The same, a score of 2; and (E) Don't know, the lowest score of 1 . The survey answers were then tabulated following the SCM procedures. In this study, four out of the 14 participants $\left(N_{2}=4\right)$, who reported the most significant overall psychosocial changes, were invited to an individual interview. The 1-h interview focused on indepth examination of the processes of their psychosocial changes. The interviews took place at each of the four participants' home to ensure privacy, convenience, and comfort. The sessions were audiotaped with the consent of the interviewees.

\section{Preliminary Findings}

Five themes emerged from the focus group interviews concerning our participants' psychosocial changes, which were then rated through a survey and further discussed in individual interviews.

Table 2 shows that the psychological changes in women 8 months into the project included: an enriched daily life, better mood, and enhanced self-confidence. Whereas changes on the social dimension included: an increased willingness to socialize and accept more mutual help. The aggregated numbers presented in the table indicate the 
Table 2 Areas of psychosocial changes $\left(N_{1}=14\right)$ among focus group participants in Zhangjiaping Village, Yingxiu Town, Wenchuan County, Sichuan Province, China

\begin{tabular}{lllllll}
\hline Dimensions & Themes & Very much (A) & Much (B) & A little bit (C) & The same (D) & Don't know (E) \\
\hline Psychological & Enriched daily life & 9 & 4 & 1 & 0 & 0 \\
& Better mood & 8 & 4 & 2 & 0 & 0 \\
& Enhanced self-confidence & 7 & 4 & 3 & 0 & 0 \\
Social & Increased socialization & 5 & 8 & 1 & 0 & 0 \\
& More mutual help & 9 & 1 & 1 & 1 & 2 \\
\hline
\end{tabular}

Table 3 In-depth survey results from the four most successful participants $\left(N_{2}=4\right)$ in Zhangjiaping Village, Yingxiu Town, Wenchuan County, Sichuan Province, China

\begin{tabular}{|c|c|c|c|c|c|}
\hline \multirow[t]{2}{*}{ Dimensions } & \multirow[t]{2}{*}{ Themes } & \multicolumn{4}{|c|}{ Participant } \\
\hline & & No. 6 & No. 10 & No. 11 & No. 12 \\
\hline \multirow[t]{3}{*}{ Psychological } & Enriched daily life & 5 & 5 & 5 & 5 \\
\hline & Better mood & 4 & 5 & 4 & 5 \\
\hline & Enhanced self-confidence & 5 & 5 & 5 & 5 \\
\hline \multirow[t]{2}{*}{ Social } & Increased socialization & 5 & 5 & 4 & 4 \\
\hline & More mutual help & 5 & 5 & 5 & 5 \\
\hline Overall score & & 24 & 25 & 23 & 24 \\
\hline
\end{tabular}

extent of change participants experienced in each psychosocial theme. It is apparent that through participating in the PCB project, a majority considered they had been psychologically fulfilled by an enriched daily life; whereas more mutual help was a notable social difference observed.

The survey results of the participants were then analyzed. The scoring on individual themes and the overall score of the successful participants $\left(N_{2}=4\right)$ are presented in Table 3. The score of 5 in the table refers to the participant's choice of "Very much," the score of 4 as "Much," 3 as "Average," 2 as "little," and 1 as "Very little."

Next, we elaborate the specific themes, using excerpts from the focus group interviews as well as interviews with the four successful cases. The aim of the individual interviews was to examine in greater details the process of psychosocial changes went through by the women who took part in the Guozhuang dance project in a post-disaster local environment in China. The content of the interviews revolved around the five themes identified in the focus group study, the data presented below are therefore structured according to the themes of: enriched daily life, better mood, enhanced self-confidence, increased socialization, and more mutual help.

\subsection{Enriched Daily Life}

The women reported that they had failed to find interesting things to do before participating in the project. Joining the dance classes and other activities had enriched their daily lives. For example, when asked to describe her usual activities, Participant 12 said, "I simply watched TV all day. When my eyes got tired and sore from watching TV, I would go to bed. Then I woke up, I did it all over again. The TV was always on."

She indicated that she was moved and motivated by the passion of the social worker who encouraged women to join the Guozhuang dance class with strong enthusiasm. Through participation, she has found a purpose and meaning in life once again, and was speaking with optimism: "I'd better go and learn since I like dancing" and "now that I have opportunities to meet with other women, to dance together and to visit places for group activities." Other than going to the dance classes almost every day, the project also empowered her to take initiatives in making positive changes in other aspects of life. "In the past, I ate dinner quite late, around 8 p.m. Knowing that I can go dancing now, I start having dinner at 6 p.m. or so, and rush out to the square to dance as soon as I finish. It also got me to think I should run errands and finish chores quickly." (Participant 12) 


\subsection{Better Mood}

The opportunity to hang out with other dance group members made the participants happier and more relaxed. In particular, the project created an outlet for them to pentup anxiety. Participant 10 reported that she could "forget all the trivialities at home during the dance class and felt destressed after chatting with other women."

With an enriched life, she found it unnecessary to vent her emotions and negativities in non-productive ways. She elaborated:

After the earthquake, I quarrelled with family members frequently. But I got so much better than that right now. Members of the dance group often help each other out and do things together, like going into the mountains, travelling, team-building, and having meetings [...] (Participant 10)

With the on-going activities, she was protected in an atmosphere where she was encouraged to interact with more people, and to share her both happy and unhappy thoughts. She quipped: "I feel I am willing and able to open my heart now. When I confide in others about what bothers me, we can laugh about it and let it go."

\subsection{Enhanced Self-Confidence}

Some participants recalled feeling shy when they first joined the group. But the longer they stayed, the more confident they become. For example, Participant 6 reflected:

In the past, I would hesitate to dance in front of my neighbors in the village, and now it no longer bothers me and I have no such concern anymore [...] The last time a few women from the township came over [to the village] and I went up to perform for an occasion.

The stage was huge and there was even a crowd, but I didn't feel nervous or uneasy at all.

It is apparent that participants have been feeling more confident upon mingling and meeting with more people, even those they were unfamiliar with. Before joining the project, Participant No. 11 seldom left home to interact with others. At most, she chatted with a few within the circle of friends. She observed the change in herself that "Whatever they [other women] chat or discuss, even I have no prior knowledge about it I feel it doesn't hurt for me to ask. But in the past, I would never speak up let alone ask comfortably upon things that I wasn't sure about.”

\subsection{Increased Socialization}

The survey results revealed that all 14 participants had become more willing to interact with people, while five of them notably indicated a contrasting experience on this regard. For instance, Participant 6 remarked that after the earthquake, she felt numb, scared, stressed, and distrustful. At that point she did not feel like reaching out or talking to anyone. She would even think that people out there are evil for reasons she could not articulate.

Since joining the dance class, she felt relieved and reassured that "it is indeed not very hard to socialize with others [...] I slowly recognize that not everyone out there are bad people; in fact, most of them are sincere and can befriend with $[\ldots]$ they care about each other and keep us all going." (Participant 6)

\subsection{More Mutual Help}

Eleven participants mentioned that the project had taught them about mutual help, particularly strengthening relationships among neighbors. Through team-building activities and her observation, Participant 11 was convinced that she was not alone to deal with the traumatic experience, and people around her were not all selfish. On the contrary, they do actually care for one another. Participant 6 illustrated the team spirit that had moved her deeply:

Recently Y's younger daughter was badly burnt by boiled water, members of the dance group decided to go to her place and take turns to care for the child every night [...] Imagine if $\mathrm{Y}$ had to do all this on her own, while her husband was away, wouldn't that have been too much and exhaustive for her? Two people each night taking turns and helping her out made it much more manageable, so we should, and we did. Without this dance group, those close to $\mathrm{Y}$ could have helped indeed, but it wouldn't be the same [...] it wouldn't be coming from a collective group.

In addition, Participant 6 noticed that neighborhood relationships had been strengthened, as "everyone participates proactively whenever there is an event or ceremony."

For example, whenever there is a wedding ceremony, we [the dance group] would get together and dance till about 9 or 10 p.m., without being requested by the organizer. Dancing is not only a good exercise, it also enables us to share and express our joy. The reason we do this is that we see the village as a whole, and we should support each other whenever they need help, and naturally others will reciprocate and do the same. It makes us feel unified. (Participant 6) 


\section{Discussion}

This study found that psychosocial capacity-building activities, in this case promoting the Guozhuang cultural dance, brought about positive psychosocial changes among a group of women in a disaster-stricken rural village in China using Miller's PCB principles.

Guozhuang dance was an invaluable cultural asset to work with individuals and groups in a post-disaster Chinese context. Its impact on the affected women and community of Zhangjiaping Village underscored the importance of considering intangible resources when designing psychosocial programs. Post-disaster interventions that build on available local cultural resources can sustain their positive effects as the resources continue to be available even after outside help has retreated and left the disaster site (Miller 2012). It is important for the social work profession to ensure that cultural competency is ethically mandated as it could potentially increase the effectiveness of interventions by integrating the clients' unique cultural assets (Jani et al. 2008). In China, social work programs based on local cultural heritage, such as traditional dance and New Year painting (nian hua), have been found to help disaster survivors and communities to increase their social, mental, and psychological capacities (Sim and Dominelli 2017).

In addition to valuing intangible local resources, PCB programs in post-disaster situations are often predicated on the reconstruction and restitution of collective life-the social threads and braids that connect people and give their lives meaning (Miller 2012). This inevitably entrusts local people who know their culture, community, and language to achieve what works for them. In this study, the facilitative role of the social worker and his team empowered the local women by creating an enabling environment for them to act independently and to motivate the local people to participate, plan, and decide for themselves in the process, which is a critical component of the rebuilding process (IASC 2007). Creating such an environment is a meaningful and sustainable part of disaster recovery because it rests on long-term, ongoing relationships with community collaborators (Pinto et al. 2008). By mobilizing village women to participate from the beginning, mediating conflicts when appeared, and, most importantly, supporting them to reach out to other community members, the social work team were proud and comfortable in handing the control of the dance group over to the village women themselves and offering them the knowledge and skills they needed to act independently (Schilderman and Lyons 2011).

Although disaster does not strike everyone in the same way, this study illustrated that it is necessary not to devalue an individual's strengths and sources of resilience in any given cultural and social contexts (Miller 2012). As in this case, it was important that post-disaster activities were built on women's resilience and adaptive capacity (Drolet et al. 2015) in order to cope with the constraints that impinged on their lives. Although women's vulnerabilities are evident in times of disaster, this project revealed the significant impact of recognizing the strength of individuals, and in encouraging women's persistence in physical activity (Lloyd and Little 2010). Women's consistent participation in Guozhuang dancing facilitated them in overcoming personal fears and gaining greater self-confidence. These positive changes contributed to the fostering of community cohesion as the organization of dance groups, communal activities, and peer-support activities were all initiated and steered by the same group of women.

This study confirmed the importance of shifting the emphasis from treating individuals to the whole of community as the fundamental units of psychosocial rebuilding after a disaster (Miller 2012; Suarez 2016). Community or social networks are important elements in the rebuilding process. Where these networks are strong, people have traditions of mutual aid and information sharing (Schilderman and Lyons 2011; Gil-Rivas and Kilmer 2016). This study suggests that the community networks in Zhangjiaping Village offered positive effects to women during disaster recovery because they were therapeutic in nature. They empowered the affected across a multiplicity of life domains, including livelihood, physical health, mental health, social and cultural life, and spirituality (Ganapati 2012; Vindevogel et al. 2015).

In addition, this study provided an alternative approach to service evaluation for the social work profession working in humanitarian contexts. The research design and the corresponding methods (focus groups, surveys, and indepth interviews) used in this study were cost-effective and relatively easy to implement. Social work practitioners in China could consider utilizing them in an adaptive manner, as few are well-trained in conducting research, and, most of all, they lack the skills, support, and time to carry out quasi-experimental designs, randomized controlled trials, or correlation analyses (Sim and Lau 2016).

Nevertheless, this study is not without flaws. Specifically, there is a limited generalizability of the findings as the sample only comprised 14 women who were all participants in the PCB project from one village. This smallscale study have inevitably excluded the views of other women who did not participate in the cultural dance group. The lack of participation may include those who were unable to join owing to poor psychosocial well-being, those who thought their well-being was good enough, or those who had improved without participating in the dance group. Finally, this study drew only on participants' selfreported changes, which might have restricted the range of 
emerging themes and the depth of individual stories. Future studies could include the accounts of other stakeholders, such as family members, relatives, and neighbors.

\section{Conclusion}

This study adds to the extant literature on the role of psychosocial services in developing individual and community resilience after disasters. It has extensively described a psychosocial capacity-building project that aimed to improve the psychosocial well-being of women who were affected by the 2008 Wenchuan Earthquake. An adjusted Success Case Method was employed to examine changes on the psychological and social dimensions experienced by those who participated in a cultural dance project over the course of 8 months. However, it is necessary to note that the findings of this study reflected only the short-term effects of the project as the evaluation was conducted before the completion of it. Given the positive response by our participants, and the consideration that debris flows will continue to affect lives of people in the village, medium- to long-term observations of changes on specific groups, notably women, children, and the elderly, in the epicenter is deemed important. A comparative study with similar contexts, such as another village in Beichuan County, that was also adversely affected by the Wenchuan Earthquake, Ya'an Earthquake in Sichuan Province that occurred in 2013, or the 2014 Ludian Earthquake in Yunnan Province that affected many ethnic minority groups, could be considered. Collaborative efforts of researchers and practitioners in China and other countries, and the greater investment of resources and attention given to women disaster survivors is urgent and necessary to (1) provide more empirical studies on a group that is often neglected in research, and (2) examine and advance the argument that, when given the resources and power, women can play a leadership role to build disaster resilience even in rural, underdeveloped contexts.

Nevertheless, this study highlighted the importance of promoting culturally relevant and empowering practices for women, without undermining their resilience, as well as their significant contributions to their families and communities following disasters. The structure of this project could serve as a template for future PCB programs, where practitioners from other countries could adapt the methodology in a culturally appropriate manner and consider the use of SCM in monitoring changes throughout the process. The research limitations of this project can also be amended in future program designs. This study can be a reference point in the use of psychosocial approaches that improve disaster survivors' mental health and psychosocial well-being in post-disaster contexts. More rigorous research, however, is needed to evaluate the effectiveness of such efforts.

Acknowledgements This research was funded by The Hong Kong Polytechnic University for the project Coping with Loss in a Chinese Post-disaster Context: Comparison Case Studies in Wenchuan Yingxiu Primary School and Mianzhu Zhongxin Friendship Primary School of Sichuan (A-PM12). We thank our co-researchers from Zhangjiaping Village of Yingxiu Town for their kind support and understanding of our work.

Open Access This article is distributed under the terms of the Creative Commons Attribution 4.0 International License (http://crea tivecommons.org/licenses/by/4.0/), which permits unrestricted use, distribution, and reproduction in any medium, provided you give appropriate credit to the original author(s) and the source, provide a link to the Creative Commons license, and indicate if changes were made.

\section{References}

Aryal, K. 2014. Women's empowerment in building disaster resilient communities. Asian Journal of Women's Studies 20(1): 164-174.

Baker, L.R., and L.A. Cormier. 2014. Disasters and vulnerable populations: Evidence-based practice for the helping professions. New York: Springer.

Becker, S.M. 2007. Psychosocial care for adult and child survivors of the tsunami disaster in India. Journal of Child and Adolescent Psychiatric Nursing 20(3): 148-155.

Bonebright, D.A. 2010. 40 years of storming: A historical review of Tuckman's model of small group development. Human Resource Development International 13(1): 111-120.

Braaten, L.J. 1974/1975. Developmental phases of encounter groups and related intensive groups: A critical review of models and a new proposal. Interpersonal Development 5: 112-129.

Brinkerhoff, R.O. 2005. The success case method: A strategic evaluation approach to increasing the value and effect of training. Advances in Developing Human Resources 7(1): 86-101.

Carron, A.V., L.R. Brawley, and W.N. Widmeyer. 1998. The measurement of cohesiveness in sport groups. In Advances in sport and exercise psychology measurement, ed. J.L. Duda, 213-226. Morgantown, WV: Fitness Information Technology.

Coryn, C.L., D.C. Schröter, and C.E. Hanssen. 2009. Adding a timeseries design element to the success case method to improve methodological rigor: An application for non-profit program evaluation. American Journal of Evaluation 30(1): 80-92.

Cui, K. 2015. The insider-outsider role of a Chinese researcher doing fieldwork in China: The implications of cultural context. Qualitative Social Work 14(3): 356-369.

Cutter, S. 1996. Vulnerability to environmental hazards. Progress in Human Geography 20(4): 529-539.

Drolet, J., L. Dominelli, M. Alston, R. Ersing, G. Mathbor, and H. Wu. 2015. Women rebuilding lives post-disaster: Innovative community practices for building resilience and promoting sustainable development. Gender \& Development 23(3): 433-448.

Ganapati, N. 2012. In good company: Why social capital matters for women during disaster recovery. Public Administration Review 72(3): 419-427.

Ge, Y., P. Cui, J. Zhang, C. Zeng, and F. Su. 2015. Catastrophic debris flows on July 10th 2013 along the Min River in areas 
seriously-hit by the Wenchuan Earthquake. Journal of Mountain Science 12(1): 186-206.

Gil-Rivas, V., and R. Kilmer. 2016. Building community capacity and fostering disaster resilience. Journal of Clinical Psychology 72(12): 1318-1332.

Han, L., J. Berry, and Y. Zheng. 2016. The relationship of acculturation strategies to resilience: The moderating impact of social support among Qiang ethnicity following the 2008 Chinese earthquake. PLOS ONE 11(10): Article e0164484.

$\mathrm{Hu}$, Y. 2010. Social work of disaster based on gender: Take Wenchuan earthquake for example. Journal of Women's Academy at Shandong 93(5): 28-33 (in Chinese).

$\mathrm{Hu}$, M. 2015. Challenges of conducting disaster research: The case of the Sichuan earthquake. Risk, Hazards \& Crisis in Public Policy 6(2): 164-182.

Huang, R., and W. Li. 2014. Post-earthquake landsliding and longterm impacts in the Wenchuan Earthquake area, China. Engineering Geology 182(PB): 111-120.

Huang, Y., and H. Wong. 2013. Effects of social group work with survivors of the Wenchuan earthquake in a transitional community. Health \& Social Care in the Community 21(3): 327-337.

IASC (Inter-Agency Standing Committee). 2007. IASC guidelines on mental health and psychosocial support in emergency settings. Geneva: IASC.

Jani, J.S., L. Ortiz, and M.P. Aranda. 2008. Latino outcome studies in social work: A review of the literature. Research on Social Work Practice 19(2): 179-194.

Kibel, B.M. 1999. Success stories as hard data: An introduction to results mapping. New York: Plenum.

Leedy, P., and J.E. Ormrod. 2005. Practical research, 8th edn. Mahwah, NJ: Pearson.

Lewis, S.D., V.R. Johnson, R.P. Farris, and J.C. Will. 2004. Using success stories to share knowledge and lessons learned in health promotion. Journal of Women's Health 13(5): 616-624.

Li, J. 2007. The impact of Tibetan Guozhuang dance on the healthrelated physical fitness of senior women living in Xining region. Journal of High Altitude Medicine 17(4): 44-45.

Li, X., S. Li, and L. Qin. 2011. Influence of earthquake on menstrual cycles and psychological well-being in women who lost their children in the quake and intended to conceive again. Journal of Practical Obstetrics \& Gynecology 27(1): 69-72.

Liu, A. 2009. China reports 88,928 deaths in natural disasters last year. http://english.cctv.com/20090525/102792.shtml. Accessed 25 Mar 2019.

Liu, W., and W. Chen. 2010. Formation and evolution of sports Guozhuang for Zang and Qiang nationalities. Journal of Chengdu Sport University 36(8): 27-30 (in Chinese).

Liu, W., H. Xiu, and T. Wu. 2009. Exploring the concept of ZangQiang Guozhuang. Journal of Aba Teachers College 26(4): 106-108.

Lloyd, K., and D. Little. 2010. Keeping women active: An examination of the impacts of self-efficacy, intrinsic motivation, and leadership on women's persistence in physical activity. Women \& Health 50(7): 652-669.

Miller, J.L. 2012. Psychosocial capacity building in response to disasters. New York: Columbia University Press.

Olson, R.S. 2000. Toward a politics of disaster: Losses, values, agendas, and blame. International Journal of Mass Emergencies and Disasters 18(2): 265-287.

Pinto, R., M. Mckay, and C. Escobar. 2008. "You've gotta know the community": Minority women make recommendations about community-focused health research. Women \& Health 47(1): 83-104.

Powell, T., and S. Leytham. 2014. Building resilience after a natural disaster: An evaluation of a parental psycho-educational curriculum. Australian Social Work 67(2): 285-296.
Rao, L.L., R. Han, X.P. Ren, X.W. Bai, R. Zheng, H. Liu, Z.J. Wang, J.Z. Li, et al. 2011. Disadvantage and prosocial behavior: The effects of the Wenchuan earthquake. Evolution and Human Behavior 32(1): 63-69.

Schilderman, T., and M. Lyons. 2011. Resilient dwellings or resilient people? Towards people-centred reconstruction. Environmental Hazards 10(3-4): 218-231.

Scott, S.A., and S. Proescholdbell. 2009. Informing best practice with community practice: The community change chronicle method for program documentation and evaluation. Health Promotion Practice 10(1): 102-110.

Shenton, A.K. 2004. Strategies for ensuring trustworthiness in qualitative research projects. Education for Information 22(2): 63-75.

Sim, T. 2009. Crossing the river stone by stone: Developing an expanded school mental health network in post-quake Sichuan, China. Journal of Social Work 2(3): 165-177.

Sim, T, and L. Dominelli. 2017. When the mountains move: A Chinese post-disaster psychosocial social work model. Qualitative Social Work 16(5): 594-611.

Sim, T., and V.C. Lau. 2016. The emergence of social work practice research in the Peoples' Republic of China: A literature review. Research on Social Work Practice 27(1): 8-18.

Siu, P. 2016. Eight years on, Sichuan earthquake survivors remember. The South China Morning Post. 12 May 2016. https://www. scmp.com/news/hong-kong/health-environment/article/1944085/ eight-years-sichuan-earthquake-survivors-remember. Accessed 19 Apr 2019.

Sohrabizadeh, S., S. Tourani, and H.R. Khankeh. 2016 Women and health consequences of natural disasters: Challenge or opportunity? Women \& Health 56(8): 977-993.

Suarez, E.B. 2016. Trauma in global contexts: Integrating local practices and socio-cultural meanings into new explanatory frameworks of trauma. International Social Work 59(1): 141-153.

Tang, C., J. Zhu, X. Qi, and J. Ding. 2011. Landslides induced by the Wenchuan Earthquake and the subsequent strong rainfall event: A case study in the Beichuan area of China. Engineering Geology 122(1-2): 22-33.

Tierney, K.J. 2007. From the margins to the mainstream? Disaster research at the crossroads. Annual Review of Sociology 33(1): 503-525.

Tol, W.A., and M. van Ommeren. 2012. Evidence-based mental health and psychosocial support in humanitarian settings: Gaps and opportunities. Evidence Based Mental Health 15(2): 25-26.

Tuckman, B.W., and M.A. Jensen. 1977. Stages of small-group development revisited. Group and Organization Studies 2(4): 419-427.

Twigg, J. 2004. Disaster risk reduction: Mitigation and preparedness in development and emergency programming. London: Humanitarian Practice Network, Overseas Development Institute.

Vindevogel, S., A. Ager, J. Schiltz, E. Broekaert, and I. Derluyn. 2015. Toward a culturally sensitive conceptualization of resilience: Participatory research with war-affected communities in northern Uganda. Transcultural Psychiatry 52(3): 396-416.

Wisner, B., P. Blaikie, T. Cannon, and I. Davis. 2004. At risk: Natural hazards, people's vulnerability and disaster, 2nd edn. New York: Routledge.

Xu, Q., S. Zhang, W. Li, and T. van Asch. 2012. The 13 August 2010 catastrophic debris flows after the 2008 Wenchuan Earthquake, China. Natural Hazards and Earth System Sciences 12(1): 201-216.

Zhang, M., and L.X. Liu. 2014. Research on the impact of Guozhuang dance on mental health rehabilitation. Education Teaching Forum 23: 151-152. 